\title{
Stage III Renal Pelvis and Ureter Cancer AJCC v7
}

National Cancer Institute

\section{Source}

National Cancer Institute. Stage III Renal Pelvis and Ureter Cancer A/CC v7. NCI

Thesaurus. Code C7521.

Stage III includes: T3, NO, M0. T3: For renal pelvis only: tumor invades beyond muscularis into peripelvic fat or the renal parenchyma. For ureter only: tumor invades beyond muscularis into periureteric fat. NO: No regional lymph node metastasis. MO: No distant metastasis. (AJCC 7th ed.) 